\title{
Thermo-electric properties of quantum point contacts
}

\author{
H van Houtent, L W Molenkampt, C W J Beenakkert and \\ C T Foxon $\ddagger$
}

†Philips Research Laboratories, 5600 JA Eindhoven, The Netherlands

‡Philips Research Laboratories, Redhill, Surrey RH1 5HA, UK

\begin{abstract}
The conductance, the thermal conductance, the thermopower and the Peltier coefficient of a quantum point contact all exhibit quantum size effects. We review and extend the theory of these effects. In addition, we review our experimental work on the quantum oscillations in the thermopower, observed using a current heating technique. New data are presented showing evidence for quantum steps in the thermal conductance, and (less unequivocally) for quantum oscillations in the Peltier coefficient. For these new experiments we have used a quantum point contact as a miniature thermometer.
\end{abstract}

\section{Introduction}

A quantum point contact is a short constriction of variable width, comparable to the Fermi wavelength, defined using a split-gate technique in a high-mobility two-dimensional electron gas (2DEG). Quantum point contacts $[1,2]$ are best known for their quantized conductance at integer multiples of $2 e^{2} / h$. For a general review of quantum transport in semiconductor nanostructures see [3]. The thermo-electric properties of quantum point contacts have recently begun to be explored as well.

The Landauer-Büttiker formalism $[4,5]$, which treats electrical transport as a transmission problem between reservoirs, has been generalized to thermal transport and to thermo-electric cross-effects by Sivan and Imry [6] and by Butcher [7]. Streda [8] has considered the specific problem of the thermopower $S$ of a quantum point contact. He found that $S$ vanishes whenever the conductance of the point contact is quantized, and that it exhibits peaks between quantized conductance plateaux. The magnitude of the peaks depends on the energy dependence of the transmission probability $t(E)$ through the point contact. To the extent that a quantum point contact behaves like an ideal electron waveguide, $t(E)$ has a unit step-function energy dependence. A somewhat more realistic model of a quantum point contact - introduced by Büttiker [9]-is to assume that the electrostatic potential has a saddle shape. This particular model has also been used to calculate the thermopower [10]. The same theoretical framework can be used to evaluate the thermal conductance $\kappa$ and the Peltier coefficient $\Pi$, which exhibit quantum size effects similar to those in the conductance and the thermopower, respectively. We review the theory in section 2. For a discussion of thermo-electric effects in different transport regimes, we refer to a recent article by Ben-Jacob et al [11].
We have used a current heating technique $\dagger$ to observe the characteristic quantum size effects in the thermo-electric properties of a quantum point contact. Our previous work on the quantum oscillations in the thermopower $S[13,14]$ is reviewed in subsection 3.1 . Because of the sizable thermopower, a quantum point contact can be used as a miniature 'thermometer', to probe the local temperature of the electron gas. We have exploited this in our design of novel devices with multiple quantum point contacts, with which we demonstrate quantum steps in the thermal conductance $\kappa$ as well as quantum oscillations in the Peltier coefficient of a quantum point contact. The first results of these experiments are presented in subsections 3.2 and 3.3. Concluding remarks are given in section 4.

\section{Theoretical background}

\subsection{Landauer-Büttiker formalism of thermo-electricity}

The Landauer-Büttiker formalism $[4,5]$ relates the transport properties of a conductor to the transmission probabilities between reservoirs that are in local equilibrium. Let us assume that only two such reservoirs are present. In equilibrium, the reservoirs are at chemical potential $E_{\mathrm{F}}$ and temperature $T$. In the regime of linear response, the current $I$ and heat flow $Q$ are related to the chemical potential difference $\Delta \mu$ and the temperature difference $\Delta T$ by the constitutive equations [15]

$$
\left(\begin{array}{l}
I \\
Q
\end{array}\right)=\left(\begin{array}{cc}
G & L \\
M & K
\end{array}\right)\left(\begin{array}{c}
\Delta \mu / e \\
\Delta T
\end{array}\right)
$$

The thermo-electric coefficients $L$ and $M$ are related by

† Current heatıng has also been used by Gallagher et al [12] to study fluctuations in the thermopower in the phase coherent diffusive transport regıme. 
an Onsager relation, which in the absence of a magnetic field is

$$
M=-L T .
$$

Equation (1) is often re-expressed with the current $I$ rather than the electrochemical potential $\Delta \mu$ as an independent variable [15],

$$
\left(\begin{array}{c}
\Delta \mu / e \\
Q
\end{array}\right)=\left(\begin{array}{cc}
R & S \\
\Pi & -\kappa
\end{array}\right)\left(\begin{array}{c}
I \\
\Delta T
\end{array}\right)
$$

The resistance $R$ is the reciprocal of the isothermal conductance $G$. The thermopower $S$ is defined as

$$
S \equiv\left(\frac{\Delta \mu / e}{\Delta T}\right)_{I=0}=-L / G
$$

The Peltier coefficient $\Pi$, defined as

$$
\Pi \equiv\left(\frac{Q}{I}\right)_{\Delta T=0}=M / G=S T
$$

is proportional to the thermopower $S$ in view of the Onsager relation (2). Finally, the thermal conductance $\kappa$ is defined as

$$
\kappa \equiv-\left(\frac{Q}{\Delta T}\right)_{I=0}=-K\left(1+\frac{S^{2} G T}{K}\right) .
$$

The thermo-electric coefficients are given in the Landauer-Büttiker formalism by $[6,7]$

$$
\begin{aligned}
G & =-\frac{2 e^{2}}{h} \int_{0}^{\infty} \mathrm{d} E \frac{\partial f}{\partial E} t(E) \\
L & =-\frac{2 e^{2}}{h} \frac{k_{\mathrm{B}}}{e} \int_{0}^{\infty} \mathrm{d} E \frac{\partial f}{\partial E} t(E)\left(E-E_{\mathrm{F}}\right) / k_{\mathrm{B}} T \\
\frac{K}{T} & =\frac{2 e^{2}}{h}\left(\frac{k_{\mathrm{B}}}{e}\right)^{2} \int_{0}^{\infty} \mathrm{d} E \frac{\partial f}{\partial E} t(E)\left[\left(E-E_{\mathrm{F}}\right) / k_{\mathrm{B}} T\right]^{2} .
\end{aligned}
$$

These integrals are convolutions of $t(E)$, which characterizes the conductor, and a kernel of the form $\varepsilon^{m} \mathrm{~d} f / \mathrm{d} \varepsilon$, $m=0,1,2$, with $\varepsilon \equiv\left(E-E_{\mathrm{F}}\right) / k_{\mathrm{B}} T$, and $f$ the Fermi function

$$
f(\varepsilon)=[\exp (\varepsilon)+1]^{-1} .
$$

Plots of these kernals are given in figure 1 .

Both $\mathrm{d} f / \mathrm{d} \varepsilon$ and $\varepsilon^{2} \mathrm{~d} f / \mathrm{d} \varepsilon$ are symmetric functions of $\varepsilon$, which is why the conductance, $G$, and the thermal conductances $K$ and $\kappa$ are determined to first order by $t\left(E_{\mathrm{F}}\right)$. (The term within brackets in equation (6) is usually small.) In contrast, $\varepsilon \mathrm{d} f / \mathrm{d} \varepsilon$ is an anti-symmetric function of $\varepsilon$, so that the thermo-electric cross-coefficients $L, S, M$, and $\Pi$ are determined mainly by the derivative $\mathrm{d} t(E) / \mathrm{d} E$ at $E=E_{\mathrm{F}}$. This is substantiated by a Sommerfeld expansion of the integrals (7)-(9), valid for a smooth function $t(E)$ to lowest order in $k_{\mathrm{B}} T / E_{\mathrm{F}}[7]$

$$
\begin{aligned}
& G \approx \frac{2 e^{2}}{h} t\left(E_{\mathrm{F}}\right) \\
& L \approx \frac{2 e^{2}}{h} L_{0} e T\left(\frac{\mathrm{d} t(E)}{\mathrm{d} E}\right)_{E=E_{\mathrm{F}}}
\end{aligned}
$$

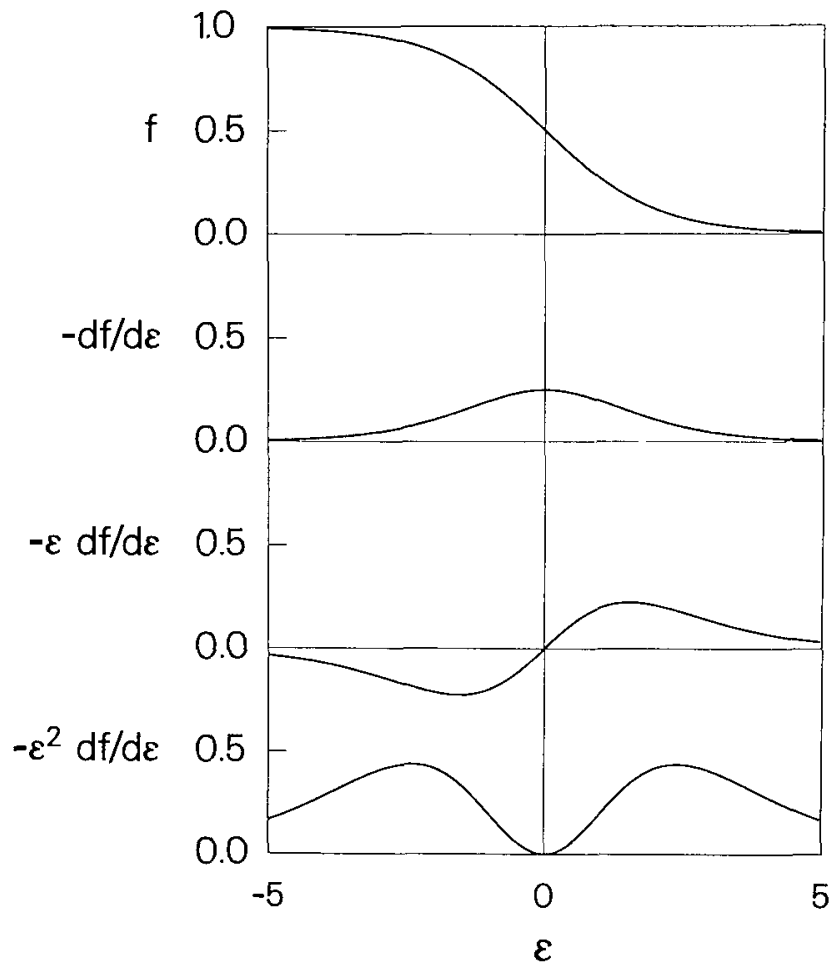

Figure 1. From top to bottom: Fermi-Dirac distribution function $f$, and $\varepsilon^{m} \mathrm{~d} f / \mathrm{d} \varepsilon$, for $m=0,1,2$, as a function of $\varepsilon \equiv\left(E-E_{\mathrm{F}}\right) / k_{\mathrm{B}} T$. These functions appear in expressions (7) - (9) for the thermo-electric coefficients.

$$
K \approx-\frac{2 e^{2}}{h} L_{0} T t\left(E_{\mathrm{F}}\right)
$$

with $L_{0} \equiv\left(k_{\mathrm{B}} / e\right)^{2} \pi^{2} / 3$ the Lorentz number. In this approximation $K=-L_{0} T G$, so that for $S^{2} \ll L_{0}$ one finds from (6) the Wiedemann-Franz relation

$$
\kappa \approx L_{0} T G .
$$

As discussed below, the thermo-electric coefficients of a quantum point contact may exhibit significant deviations from equations (11)-(14). The inadequacy of the Sommerfeld expansion is a consequence of the strong energy dependence of $t(E)$ near $E_{\mathrm{F}}$. In addition, $S^{2} \ll L_{0}$ does not hold for a quantum point contact close to pinch-off.

\subsection{Quantum point contacts as ideal electron waveguides}

In this subsection we discuss the thermo-electric properties of a quantum point contact modelled as an ideal electron waveguide, matched perfectly to the reservoirs at entrance and exit. Such a waveguide has a transmission probability with step-function energy dependence

$$
t(E)=\sum_{n=1}^{\infty} \theta\left(E-E_{n}\right) .
$$

The steps in $t(E)$ coincide with the threshold energies $E_{n}$ of the one-dimensional subbands or modes in the quantum point contact. The integrals over the energy (7) and (8) determining the conductance and the thermo- 
power can be evaluated analytically. By substitution of (15) into (7), one finds for the conductance

$$
G=\frac{2 e^{2}}{h} \sum_{n=1}^{\infty} f\left(\varepsilon_{n}\right)
$$

with $\varepsilon_{n} \equiv\left(E_{n}-E_{\mathrm{F}}\right) / k_{\mathrm{B}} T$. This reduces to $G=\left(2 e^{2} / h\right) N$ at low temperatures ( $N$ is the number of occupied subbands). Similarly, using the identity

$$
\int_{0}^{\infty} f \mathrm{~d} E=k_{\mathrm{B}} T \ln \left[1+\exp \left(E_{\mathrm{F}} / k_{\mathrm{B}} T\right)\right]
$$

we find the exact result

$$
L=\frac{2 e^{2}}{h} \frac{k_{\mathrm{B}}}{e} \sum_{n=1}^{\infty}\left[\ln \left(1+\mathrm{e}^{-\varepsilon_{n}}\right)+\varepsilon_{n}\left(1+\mathrm{e}^{\varepsilon_{n}}\right)^{-1}\right] .
$$

The thermopower $S=-L / G$ and the Peltier coefficient $\Pi=T S$ follow immediately from (16) and (18). At low temperatures the thermopower vanishes, unless the Fermi energy is withın $k_{\mathrm{B}} T$ from a subband bottom. In the limit $T=0$ one finds from (16), (18) that the maxima are given by

$$
S=-\frac{k_{\mathrm{B}}}{e} \frac{\ln 2}{N-\frac{1}{2}} \quad \text { if } E_{\mathrm{F}}=E_{N} ; N>1 .
$$

(Note that at $E_{\mathrm{F}}=E_{N}$ one also has $G=\left(2 e^{2} / h\right)\left(N-\frac{1}{2}\right)$.) Equation (19) was first obtained by Streda [8]. For the step-function model the width of the peaks in the thermopower as a function of $E_{\mathrm{F}}$ is of order $k_{\mathrm{B}} T$, at least in the linear transport regime of small applied temperature differences across the point contact $(\Delta T \ll T)$.

The thermopower of a quantum point contact with a step-function $t(E)$ does not exhibit a peak near $E_{\mathrm{F}}=E_{1}$. Instead, it follows from (16) and (18) that $-S$ increases monotonically as $E_{\mathrm{F}}$ is reduced below $E_{1}$

$$
S \approx-\frac{k_{\mathrm{B}}}{e}\left(1+\varepsilon_{1}\right)
$$

Note also that for $\varepsilon_{1} \gg 1, S$ increases as $1 / T$ as the temperature is reduced. This result is probably not very realistic. Indeed, for a saddle-shaped potential model of a quantum point contact we find instead in this regime a constant value which is proportional to $T$ (see subsection 2.3).

Plots of the thermo-electric coefficients as a function of Fermi energy, calculated from (7)-(9) and (15), are given in figures $2(a)$ and $2(b)$, for $T=1 \mathrm{~K}$ and $T=4 \mathrm{~K}$ respectively. The values for $E_{n}$ are those for a parabolic lateral confinement potential $V(y)=V_{0}+\frac{1}{2} m \omega_{y}^{2} y^{2}$, with $\hbar \omega_{y}=2.0 \mathrm{meV}$. We draw the following conclusions from these calculations.

1. The temperature $T$ affects primarily the width of the steps in $G$, and of the peaks in $S$, leaving the value of $G$ on the plateaux, and the height of the peaks in $S$ essentially unaffected.

2. The thermal conductance $\kappa$ (divided by $L_{0} T$ ) exhibits secondary plateaux near the steps in $G$, in violation of the Wiedemann-Franz law. At $4 \mathrm{~K}$ the secondary plateaux in $\kappa$ are even more pronounced than those in phase with
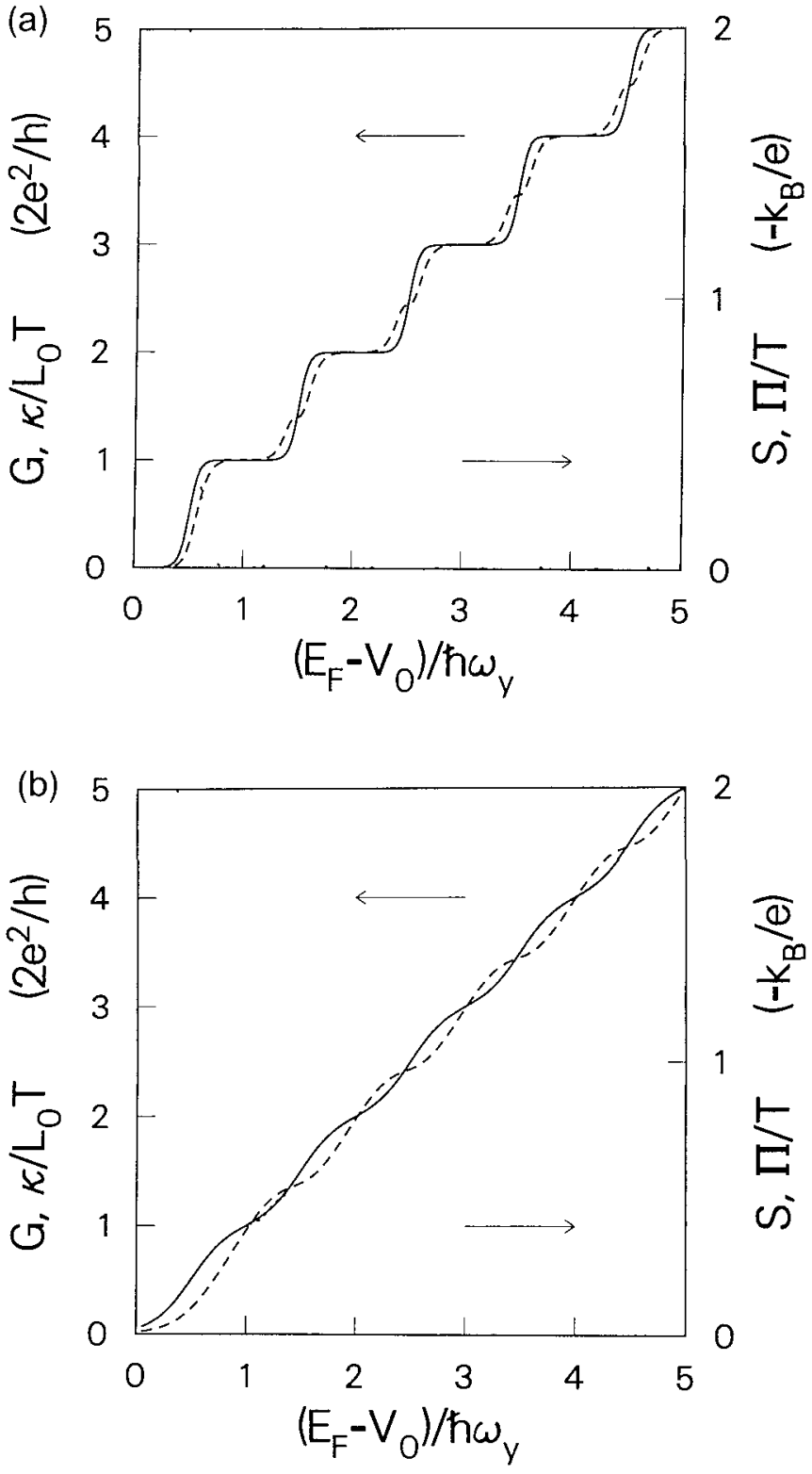

Figure 2. Calculated conductance $G$ (full curve), thermal conductance $\kappa / L_{0} T$ (broken curve), and the thermopower $S$ and Peltier coefficient $\Pi / T=S$ (same dotted curve) for a quantum point contact with step function $t(E)$ as a function of Fermı energy at (a) $1 \mathrm{~K}$ and $(b) 4 \mathrm{~K}$ The parameter used in the calculation is $\hbar \omega_{y}=2 \mathrm{meV}$.

the plateaux in the conductance. These plateaux, which apparently have not been noted previously, are due to the bimodal shape of the kernel $\varepsilon^{2} \mathrm{~d} f / \mathrm{d} \varepsilon$ (see figure 1).

3. The coefficients $\kappa$ and $K$ differ from each other whenever the thermopower $S$ does not vanish (cf (6)). We have verified that this correction is usually negligible, except in the vicinity of the first step in $G$.

\subsection{Saddle-shaped potential}

A more realstic model of a quantum point contact should account for the rounding of the steps in $t(E)$. One way to do this is to model the electrostatic potential 
$V(x, y)$ in the quantum point contact by a saddle-shaped function [9]

$$
V(x, y)=V_{0}-\frac{1}{2} m \omega_{x}^{2} x^{2}+\frac{1}{2} m \omega_{y}^{2} y^{2}
$$

where $V_{0}$ is the height of the saddle, $\omega_{x}$ characterizes the curvature of the potential barrier in the constriction, and $\omega_{y}$ the lateral confinement. The energies $E_{n}$ are given by

$$
E_{n}=V_{0}+\left(n-\frac{1}{2}\right) \hbar \omega_{y} .
$$

The transmission probability is $[16]$

$$
t(E)=\sum_{n=1}^{\infty}\left[1+\exp \left(\frac{-2 \pi\left(E-E_{n}\right)}{\hbar \omega_{x}}\right)\right]^{-1} .
$$

Note that the step-function $t(E)$ is recovered in the limit $\omega_{x} / \omega_{y} \rightarrow 0$.

To allow a comparison with the results in figure 2 for the step-function transmission probability, we have calculated the thermo-electric coefficients as a function of Fermi energy from (7)-(9) and (23), using the same value of $2 \mathrm{meV}$ for the subband separation $\hbar \omega_{y}$, and taking $\hbar \omega_{x} \approx 0.8 \mathrm{meV}$ in order to reproduce the typically observed conductance step-widths at low temperatures. The results at $T=4 \mathrm{~K}$ (not shown) were found to be identical to those given in figure $2(b)$ for the step-function $t(E)$. At $T=1 \mathrm{~K}$ there are some differences, however, as seen in figure 3 :

1. The peak heights of the oscllations in the thermopower $S$ (or in the Peltier coefficient $\Pi$ ) are reduced by about a factor of two.

2. The deviations from the Wiedemann-Franz law $\kappa=L_{0} T G$ are much smaller. In particular, the secondary plateau-like features (coinciding with the steps in $G$ ) are absent.

The behaviour of $S$ for $E_{\mathrm{F}} \ll E_{1}$ at low temperatures is qualitatively different from that discussed in subsection 2.2 for a step-function $t(E)$. Approximating $t(E) \approx\left[1+\exp \left(2 \pi\left(E_{1}-E\right) / \hbar \omega_{x}\right)\right]^{-1}$, and using the Sommerfeld expansion results (11) and (12), we find that $S$ reaches an $E_{\mathrm{F}}$-independent value (not visible in figure 3)

$$
S \approx-\frac{k_{\mathrm{B}}}{e} \frac{2 \pi^{3}}{3} \frac{k_{\mathrm{B}} T}{\hbar \omega_{x}} \quad E_{\mathrm{F}} \ll E_{1}
$$

which is proportional to $T$.

\section{Experiments}

\subsection{Thermopower}

We have previously reported $[13,14]$ the observation of quantum oscillations in the thermopower $S$ of a quantum point contact using a current heating technique. We review the main results here. The experimental arrangement is shown schematically in figure $4(a)$. By means of negatively biased split gates, a channel is defined in the 2DEG in a GaAs-AlGaAs heterostructure. A quantum point contact is incorporated in each channel boundary. The point contacts 1 and 2 face each other, so that the

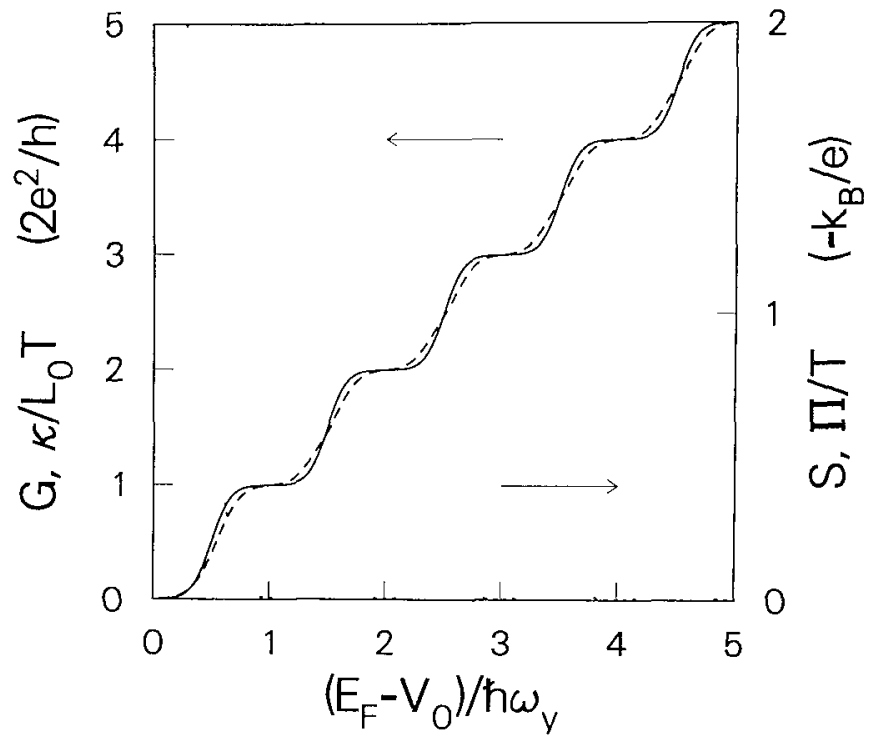

Figure 3. Calculated conductance $G$ (full curve), thermal conductance $\kappa / L_{0} T$ (broken curve), and the thermopower $S$ and Peltier coefficient $\Pi / T=S$ (same dotted curve) for a quantum point contact with a saddle shaped potential, as a function of Fermı energy at $1 \mathrm{~K}$ Parameters used in the calculation are $\hbar \omega_{y}=2 \mathrm{meV}, \hbar \omega_{x}=0.8 \mathrm{meV}$.

voltage difference $V_{1}-V_{2}$ (measured using ohmic contacts attached to the 2DEG regions behind the point contacts) does not contain a contribution from the voltage drop along the channel.

On passing a current $I$ through the channel, the average kinetic energy of the electrons increases, because of the dissipated power (equal to $\left(I / W_{\mathrm{ch}}\right)^{2} \rho$ per unit area, for a channel of width $W_{\mathrm{ch}}$ and resistivity $\rho$ ). We ignore the net drift velocity acquired by the electron gas, and assume that we can describe the non-equilibrium energy distribution in the channel by a heated Fermi function at temperature $T+\Delta T$. Since the point contacts are operated as voltage probes, drawing no net current, the temperature difference $\Delta T$ gives rise to a net thermovoltage

$$
V_{1}-V_{2}=\left(S_{1}-S_{2}\right) \Delta T
$$

As dictated by the symmetry of the channel (see figure $4(a)$ ), this voltage difference vanishes unless the point contacts are adjusted differently, so that they have unequal thermopowers $S_{1} \neq S_{2}$.

A typical experimental result [13] is shown in figure $4(b)$. The gate voltage defining point contact 1 is scanned, while that of point contact 2 is kept constant. In this way, any change in the voltage difference $V_{1}-V_{2}$ is due to variations in $S_{1}$. ( $S_{2}$ is not entirely negligible, which is why the trace for $-\left(V_{1}-V_{2}\right)$ drops below zero in figure 4(b).) Also shown is the conductance $G$ of point contact 1, obtained from a separate measurement. For more negative gate voltages, where the point contact resistance exhibits quantized plateaux, we observe strong oscillations in $V_{1}-V_{2}$. The peaks occur at gate voltages where $G$ changes stepwise because of a change in the number of occupied 1D subbands in point contact 1. These ob- 

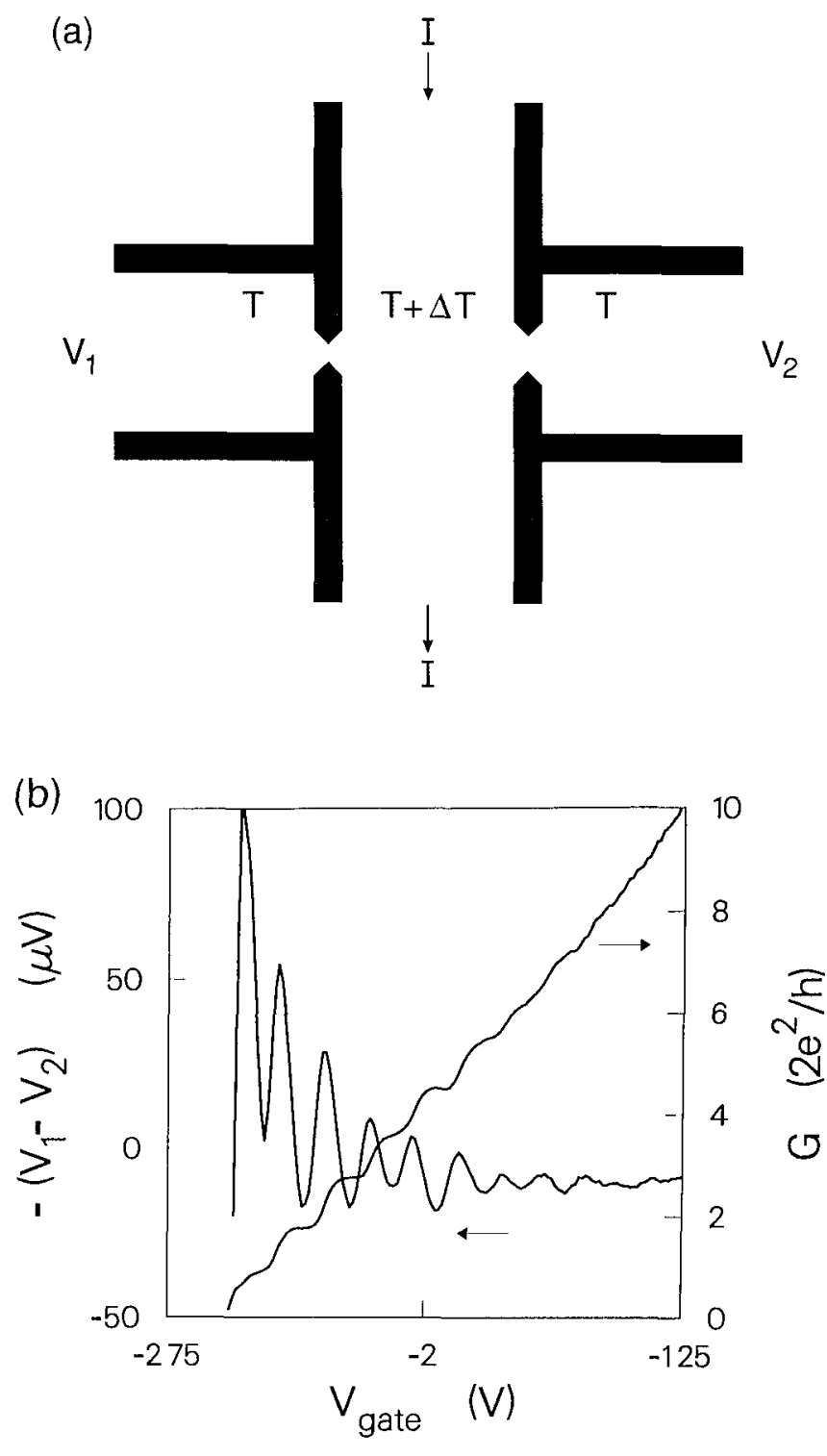

Figure 4. (a) Schematic representation of the device used to demonstrate quantum oscillations in the thermopower of a quantum point contact by means of a current heatıng technique. The channel has a width of $4 \mu \mathrm{m}$, and the two opposite quantum point contacts at its boundaries are adjusted differently. $(b)$ Measured conductance and voltage $-\left(V_{1}-V_{2}\right)$ as a function of the gate voltage definıng point contact 1 , at a lattice temperature of $165 \mathrm{~K}$ and a current of $5 \mu \mathrm{A}$. The gates defınıng point contact 2 were kept at $-2.0 \mathrm{~V}$.

servations are a manifestation of the quantum oscillations in $S$ described in section 2.

A detailed comparison of the oscillations in figure $4(b)$ with the ideal electron waveguide model (extended to the regime of finite thermovoltages and temperature differences) has been presented elsewhere [13]. The decrease in amplitude of consecutive peaks is in agreement with equation (19). We therefore only discuss the amplitude of the strong peak near $G=1.5\left(2 e^{2} / h\right)$. The stepfunction transmission probability result (19) predicts $S \sim-40 \mu \mathrm{VKK}^{-1}$ for this peak, but a value $S \sim-20 \mu \mathrm{VK}^{-1}$ is probably more realistic (cf figure 3 ). The measured value of about $50 \mu \mathrm{V}$ for the amplitude of that peak thus indicates that the temperature of the electron gas in the channel is $\Delta T \sim 2 \mathrm{~K}$ above the lattice temperature $T=1.65 \mathrm{~K}$.

The increase in temperature $\Delta T$ is expected to be related to the current in the channel by the heat balance equation

$$
c_{\mathrm{v}} \Delta T=\left(I / W_{\mathrm{ch}}\right)^{2} \rho \tau_{\varepsilon}
$$

with $c_{\mathrm{y}}=\left(\pi^{2} / 3\right)\left(k_{\mathrm{B}} T / E_{\mathrm{F}}\right) n_{\mathrm{s}} k_{\mathrm{B}}$ the heat capacity per unit area, $n_{\mathrm{s}}$ the electron density, and $\tau_{\varepsilon}$ an energy relaxation time associated with energy transfer from the electron gas to the lattice. The symmetry of the geometry implies that $V_{1}-V_{2}$ should be even in the current, and equation (26) predicts more specifically that the thermovoltage difference $V_{1}-V_{2} \propto \Delta T$ should be proportional to $I^{2}$-at least for small current densities. This is born out by experiment $[13,14]$ (not shown). Equation (26) allows us to determine the time $\tau_{\varepsilon}$ from the experimental value $\Delta T \sim 2 \mathrm{~K}$. Under the experimental conditions of figure $4(i)$ we have $T=1.65 \mathrm{~K}, I=5 \mu \mathrm{A}, W_{\text {ch }}=4 \mu \mathrm{m}, \rho=20 \Omega$. We thus find $\tau_{\varepsilon} \sim 10^{-10} \mathrm{~s}$, which is not an unreasonable value for the 2DEG in GaAs-AlGaAs heterostructures at helium temperatures [17].

The sudden decrease in $V_{1}-V_{2}$ beyond the last peak (strong negative gate voltages) is not quite understood. As discussed in section 2, the behaviour of $S$ in this regime depends crucially on the details of the energy dependence of $t(E)$.

\subsection{Thermal conductance}

The sizable thermopower of a quantum point contact (up to $-40 \mu \mathrm{V} \mathrm{K}^{-1}$ ) suggests its possible use as a miniature thermometer, suitable for local measurements of the electron gas temperature. We have used this idea in an experiment designed to demonstrate the quantum steps in the thermal conductance of a second quantum point contact.

The geometry of the device is shown schematically in figure $5(a)$. The main channel has a boundary containing a quantum point contact. Using current heating, the electron gas temperature in the channel is increased by $\Delta T$, giving rise to a heat flow $Q$ through the point contact. This causes a steady state temperature rise $\delta T$ of the 2DEG region behind the point contact (neglected in the previous subsection), which we detect by a measurement of the thermovoltage across a second point contact situated in that region.

To increase the sensitivity of our experiment, we have used a low-frequency AC current to heat the electron gas in the channel, and a lock-in detector tuned to the second harmonic to measure the root-mean-square amplitude of the thermovoltage $V_{1}-V_{2}$. The voltages on the gates defining the second quantum point contact were adjusted so that its conductance was $G=1.5\left(2 e^{2} / h\right)$. Finally, we applied a very weak magnetic field $(15 \mathrm{mT})$ to avoid detection of hot electrons on ballistic trajectories from the first to the second point contact.

Figure 5(b) shows a plot of the measured thermovoltage as a function of the voltage on the gates defining the 
(a)
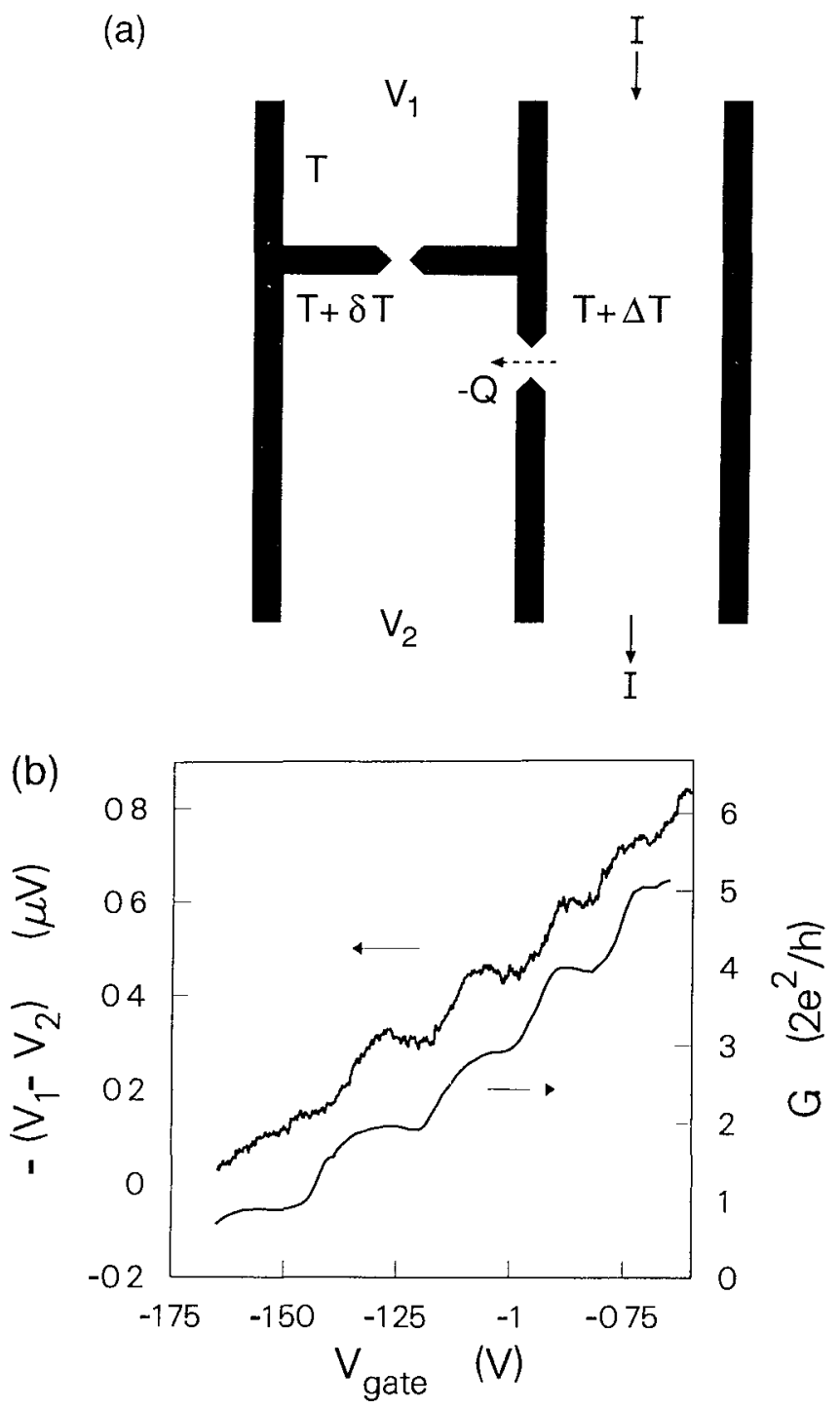

Figure 5. (a) Schematic representation of the device used to demonstrate quantum steps in the thermal conductance of a quantum point contact, using another point contact as a miniature thermometer. The main channel is $0.4 \mu \mathrm{m}$ wide. $(b)$ Measured conductance and RMS value of the second harmonic component of the voltage $V_{1}-V_{2}$ as a function of the gate voltage defining the point contact in the main channel boundary, at a lattıce temperature of $1.4 \mathrm{~K}$ and an alternating current of RMS amplitude $0.6 \mu \mathrm{A}$. The gates definıng the other point contact were kept at $-1.4 \mathrm{~V}$, so that its conductance is $G=1.5\left(2 e^{2} / h\right)$.

point contact in the channel boundary, for a channel current of $0.6 \mu \mathrm{A}$ (RMS value). A sequence of plateaux is clearly visible, lining up with the quantized conductance plateaux of the point contact. Since the measured thermovoltage is directly proportional to $\delta T$, which in turn is proportional to the heat flow $Q$ through the point contact, this result is a demonstration of the expected quantum plateaux in the thermal conductance $\kappa \equiv-Q / \Delta T$ at zero net current [ $\operatorname{cf}(6)]$. We have verified that the second-harmonic thermovoltage signal at fixed gate voltages is proportional to $I^{2}$, as expected. Let us now see whether the magnitude of the effect can be accounted for as well.
To estimate the temperature increase $\delta T$ in the region behind the point contact, we write the heat balance for that region of area $A$ (valid if $\delta T \ll \Delta T$ )

$$
\kappa \Delta T=c_{\mathrm{v}} A \delta T / \tau_{\varepsilon} .
$$

We assume that $A$ equals the square of the diffusion length $\left(D \tau_{\varepsilon}\right)^{1 / 2} \sim 10 \mu \mathrm{m}$, so that $\tau_{\varepsilon}$ drops out of (27). On inserting the Wiedemann-Franz approximation $\kappa \approx L_{0} T G$, with $G=N\left(2 e^{2} / h\right)$, and using the expression for the heat capacity per unit area given in the previous subsection (with $n_{\mathrm{s}}=E_{\mathrm{F}} m / \pi \hbar^{2}$ ), we find

$$
\frac{\delta T}{\Delta T} \approx N \frac{\hbar}{m D} \text {. }
$$

In the experiment $D=1.4 \mathrm{~m}^{2} \mathrm{~s}^{-1}$, so that at the $N=1$ plateau in the conductance, we have $\delta T / \Delta T$ $\approx 1.2 \times 10^{-3}$. The experimental curve in figure $5(b)$ was obtained at a current density in the main channel of $I / W_{\mathrm{ch}}=1.2 \mathrm{~A} \mathrm{~m}^{-1}$, nearly equal to that used in the thermopower experiment shown in figure $4(b)$. The analysis of the latter data indicated that $\Delta T \approx 2 \mathrm{~K}$ at this current density. Consequently, $\delta T \approx 2 \mathrm{mK}$. The point contact used as a thermometer (adjusted to $G=1.5\left(2 e^{2} / h\right)$ ) has $S \approx-20 \mu \mathrm{VK}^{-1}$ (see subsection 2.3 ), so that we finally obtain $V_{1}-V_{2} \approx-0.05 \mu \mathrm{V}$. The measured value is larger (cf the first plateau in figure 5(b)), but only by a factor of two. All approximations considered, this is quite satisfactory.

\subsection{Peltier effect}

In this subsection we present preliminary results of an experiment designed to observe the quantum oscillations in the Peltier coefficient $\Pi$ of a quantum point contact. The geometry of the experiment is shown schematically in figure 6(a). A main channel, defined by split gates, is separated in two parts by a barrier containing a point contact. A positive current $I$ passed through this point contact is accompanied by a negative Peltier heat flux $Q=\Pi I$, giving rise to a (steady state) temperature rise $\delta T$ in the upper part of the channel, and to a temperature drop $\delta T$ in the lower half. These temperature changes of the electron gas can be detected by measuring the thermovoltages across additional point contacts in the channel boundaries - at least in principle.

One complication is that a total power $I^{2} / G$ is dissipated due to the finite conductance $G$ of the quantum point contact in the channel. This gives rise to a temperature rise on both sides of the point contact. The dissipated power is not equally distributed among the 2DEG regions on either side, and it is precisely this imbalance which corresponds to the Peltier heat flow $\Pi I$. We wish to detect only the temperature changes $\pm \delta T$ associated with the Peltier heat flow. This is accomplished by using an AC current, and a lock-in detector tuned to the fundamental frequency to measure the components linear in $I$ of the thermovoltages $\left(V_{1}-V_{2}\right)$ and $\left(V_{3}-V_{4}\right)$. The output voltage of the lock-in detector is divided by the current, to obtain a signal linearly 
(a)
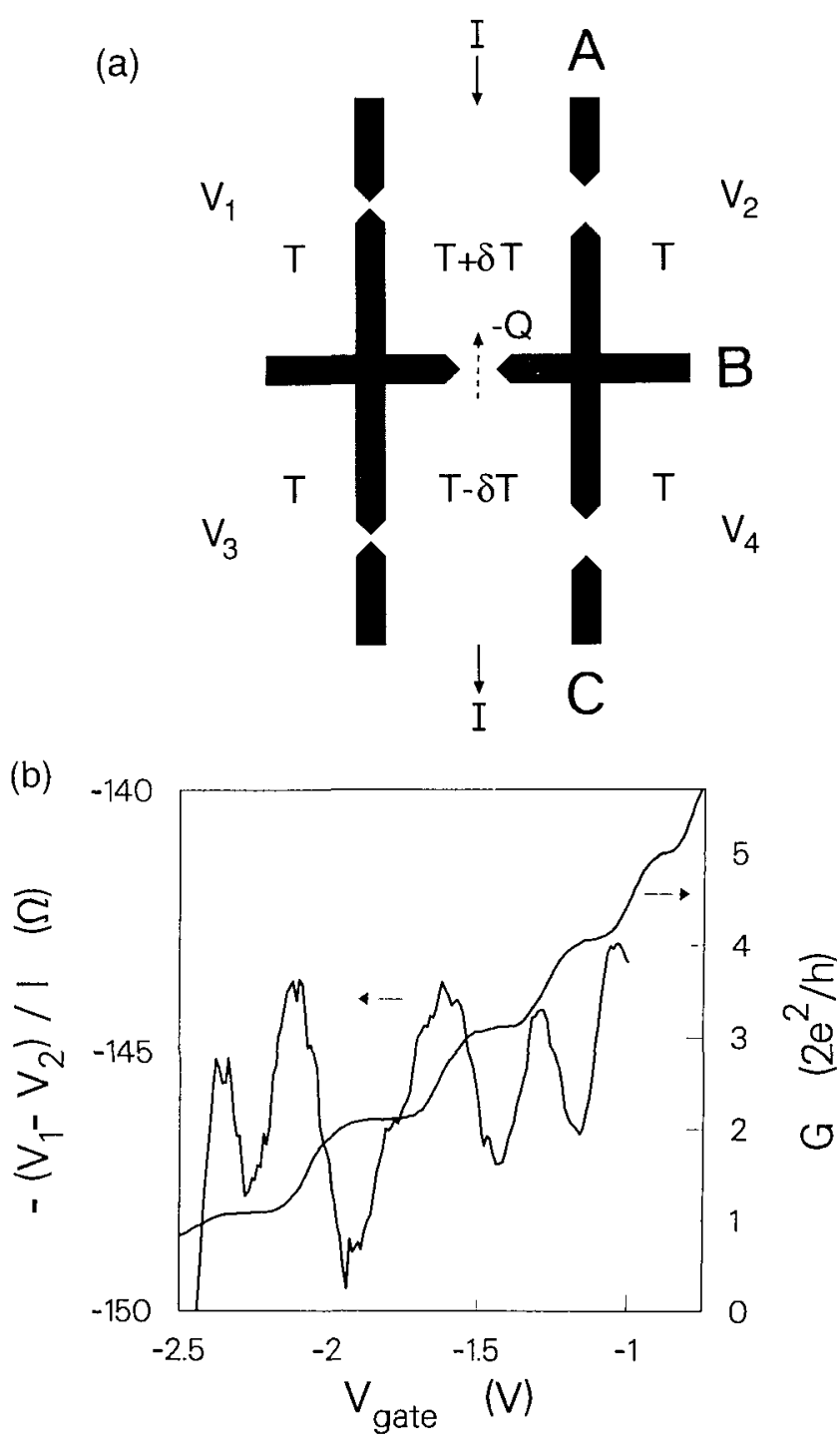

Figure 6. (a) Schematic representation of the device used to demonstrate quantum oscillations in the Peltier coefficient of a quantum point contact. Arrows indicate direction of positive flow. The main channel is $4 \mu \mathrm{m}$ wide, and the distance between the pairs of point contacts in its boundaries is $20 \mu \mathrm{m}$. (b) Measured conductance and thermovoltage $-\left(V_{1}-V_{2}\right)$ divided by the current $/$ as a function of the voltage on gate $B$, defining the point contact in the channel. The lattice temperature is $1.6 \mathrm{~K}$ and the current is about $0.1 \mu \mathrm{A}$ near $G=2 e^{2} / h$. Gates defining point contacts 1 and 3 were adjusted so that their conductance was $G=1.5\left(2 e^{2} / h\right)$. Gates A and C were unconnected.

proportional to the Peltier coefficient $\Pi$ of the point contact in the channel. This signal, measured as a function of the voltage on the gates defining that point contact, should exhibit quantum oscillations, similar to those seen in the thermopower $S$.

Unfortunately, our present sample design does not allow us to do this without also affecting the thermopower of the point contacts used as thermometers. In order to minimize this parasitic effect, we have scanned only one of the gates (labelled B in figure $6(a)$ ), and have left the adjacent gates ( $\mathrm{A}$ and $\mathrm{C}$ ), which define the reference point contacts, unconnected. The effect of gate A on the remaining two thermometer point contacts is negligible. A result obtained in this way (at $I \sim 0.1 \mu \mathrm{A}$ and at $T=1.6 \mathrm{~K}$ ) is plotted in figure $6(b)$, together with a trace of conductance versus gate voltage for the point contact in the channel.

Oscillations in $-\left(V_{1}-V_{2}\right) / I$ are clearly visible, of amplitude up to $\approx 4 \mathrm{VA}^{-1}$ and with maxima aligned with the steps between conductance plateaux. We interpret this signal as evidence for the oscillations in the Peltier coefficient $\Pi$ (see below). However, the oscillations appear to be superimposed on a much larger negative background signal. This signal (which we verified to be ohmic) is attributed to a series resistance associated with the fact that gates $\mathrm{A}$ and $\mathrm{C}$ had to be left unconnected, as mentioned above. The sum of the contact resistance at the channel exit (estimated at $\left.\left(h / 2 e^{2}\right)\left(\pi / 2 k_{\mathrm{F}} W_{\mathrm{ch}}\right) \approx 30 \Omega\right)$ and the spreading resistance associated with current flowing to the wide $2 \mathrm{DEG}$ regions of width $W_{\text {wide }} \approx 500 \mu \mathrm{m}$ (estimated at $\pi^{-1} \rho \ln \left(W_{\text {wide }} / W_{\text {ch }}\right) \approx 30 \Omega$ ) is about $60 \Omega$, which is of about the correct magnitude to be able to account for the background in figure $6(b)$. A new set of samples, designed to avoid this background signal, are currently being fabricated. (Note added in proof. Using these samples we have indeed been able to observe the quantum oscillation in $\Pi$ without such a background signal [19].)

Let us now discuss the amplitude of the oscillations in figure $6(b)$. To estimate $\delta T$, we use again the heat balance equation, and find

$$
\delta T \approx \frac{\Pi I}{c_{\mathrm{v}} D} .
$$

Using the Onsager relation $\Pi=S T$, the estimated value $S \approx-20 \mu \mathrm{V} \mathrm{K}^{-1}$ for a quantum point contact adjusted to $G=1.5\left(2 e^{2} / h\right)$, and $T=1.65 \mathrm{~K}$, we deduce $\delta T / I \approx 10^{4} \mathrm{~K} \mathrm{~A}^{-1}$. The resulting thermovoltage across one of the thermometer point contacts (adjusted to $G=1.5\left(2 e^{2} / h\right)$ as well), normalized by $I$, is about $0.3 \mathrm{~V} \mathrm{~A}^{-1}$. This is ten times smaller than the experimentally observed amplitude of the corresponding oscillation in figure $6(b)$. The origin of this discrepancy is not understood.

\section{Conclusions}

In conclusion, we have reviewed the theory of the thermo-electric effects in a quantum point contact, and our experiments on the quantum oscillations in the thermopower. New data have been presented that-for the first time-show evidence for the quantum steps in the thermal conductance, and the quantum oscillations in the Peltier coefficient. Our new experiments exploit additional quantum point contacts as miniature thermometers. We have used this technique as well in an experimental study of the effect of electron-electron scattering on the ballistic mean free path [18]. The results for the thermo-electric transport coefficients presented here compare reasonably well with the theoretical predictions. Further experiments as well as a more reliable quantitative analysis would be desirable. 


\section{Acknowledgments}

We acknowledge valuable contributions of M J P Brugmans, R Eppenga, M A A Mabesoone, $S$ van Tuinen, and Th Gravier at various stages of the experiments, and thank $\mathrm{H}$ Buyk and $\mathrm{C}$ E Timmering for their technical assistance. The support of M F H Schuurmans is gratefully acknowledged. This research was partly funded under the ESPRIT basic research action project 3133 .

\section{References}

[1] van Wees B J, van Houten H, Beenakker C W J, Williamson $\mathrm{J}$ G, Kouwenhoven L P, van der Marel $\mathrm{D}$ and Foxon C T 1988 Phys. Rev. Lett. 60848

[2] Wharam D A, Thornton T J, Newbury R, Pepper M, Ahmed H, Frost J E F, Hasko D G, Peacock D C, Ritchie D A and Jones G A C 1988 J. Phys. C: Solid State Phys. 21 L209

[3] Beenakker C W J and van Houten H 1991 Solid State Physics $\mathbf{4 4} 1$

[4] Landauer R 1957 IBM J. Res. Dev. 1223
[5] Büttiker M 1986 Phys. Rev. Lett. 571761

[6] Sivan U and Imry Y 1986 Phys. Rev. B 33551

[7] Butcher P N 1990 J. Phys. Condens. Matter 24869

[8] Streda P 1989 J. Phys. Condens. Matter 11025

[9] Büttiker M 1990 Phys. Rev. B 417906

[10] Proetto C R Preprint

[11] Ben-Jacob E, Amman M and Stern A 1991 Single Charge Tunnelling (NATO ASI Series B) ed H Grabert and M H Devoret (New York: Plenum) to be published

[12] Gallagher et al 1990 Phys. Rev. Lett. 642058

[13] Molenkamp L W, van Houten H, Beenakker C W J, Eppenga R and Foxon C T 1990 Phys. Rev. Lett. 65 1052

[14] Molenkamp L W, van Houten H, Beenakker C W J, Eppenga $\mathrm{R}$ and Foxon $\mathrm{C} \mathrm{T}$ Condensed Systems of Low Dimensionality ed J Beeby (New York: Plenum) to be published

[15] de Groot S R and Mazur P 1984 Non-Equilibrium Thermodynamics (New York: Dover)

[16] Fertig H A and Halperin B I 1987 Phys. Rev. B 36 7969

[17] Harris J J, Pals J A and Woltjer R 1989 Rep. Prog. Phys. 521217

[18] Molenkamp L W, Brugmans M J P, van Houten H and Foxon C T 1992 Semicond. Sci. Technol. 7 B 227

[19] Molenkamp L W, Gravier Th, van Houten H and Foxon C T 1992 submitted to Phys. Rev. Lett. 